\title{
Fluorescence calibration method for single-particle aerosol fluorescence instruments
}

\author{
Ellis Shipley Robinson ${ }^{1,2, a}$, Ru-Shan Gao ${ }^{1}$, Joshua P. Schwarz ${ }^{1}$, David W. Fahey ${ }^{1}$, and Anne E. Perring ${ }^{1,2}$ \\ ${ }^{1}$ NOAA Earth System Research Laboratory, Boulder, CO, USA \\ ${ }^{2}$ Cooperative Institute for Research in Environmental Sciences, Boulder, CO, USA \\ ${ }^{a}$ now at: Center for Atmospheric Particle Studies, Carnegie Mellon University, Pittsburgh, PA, USA
}

Correspondence to: Anne E. Perring (anne.perring@ noaa.gov) and E. S. Robinson (shipleyrobinson@gmail.com)

Received: 5 October 2016 - Discussion started: 7 October 2016

Revised: 7 March 2017 - Accepted: 9 March 2017 - Published: 10 May 2017

\begin{abstract}
Real-time, single-particle fluorescence instruments used to detect atmospheric bioaerosol particles are increasingly common, yet no standard fluorescence calibration method exists for this technique. This gap limits the utility of these instruments as quantitative tools and complicates comparisons between different measurement campaigns. To address this need, we have developed a method to produce size-selected particles with a known mass of fluorophore, which we use to calibrate the fluorescence detection of a Wideband Integrated Bioaerosol Sensor (WIBS-4A). We use mixed tryptophan-ammonium sulfate particles to calibrate one detector (FL1; excitation $=280 \mathrm{~nm}$, emission $=$ $310-400 \mathrm{~nm}$ ) and pure quinine particles to calibrate the other $($ FL2; excitation $=280 \mathrm{~nm}$, emission $=420-650 \mathrm{~nm})$. The relationship between fluorescence and mass for the mixed tryptophan-ammonium sulfate particles is linear, while that for the pure quinine particles is nonlinear, likely indicating that not all of the quinine mass contributes to the observed fluorescence. Nonetheless, both materials produce a repeatable response between observed fluorescence and particle mass. This procedure allows users to set the detector gains to achieve a known absolute response, calculate the limits of detection for a given instrument, improve the repeatability of the instrumental setup, and facilitate intercomparisons between different instruments. We recommend calibration of single-particle fluorescence instruments using these methods.
\end{abstract}

\section{Introduction}

Primary biological aerosol particles (PBAP) are of wide interest due to their potential impacts on air quality (e.g., Prussin II et al., 2015), ecology (e.g., Morris et al., 2013), and Earth's climate (e.g., Creamean et al., 2013). PBAP comprise a broad class of atmospheric particles ranging from the small (viruses, as small as $\sim 20 \mathrm{~nm}$ diameter) to the large (pollen grains, 5-100 $\mu \mathrm{m}$ diameter), with bacteria, fungal and plant spores, and plant, insect, and animal fragments in between (Després et al., 2012). Despite the ubiquity of PBAP, many important questions remain regarding their atmospheric impacts.

The measurement of atmospheric PBAP has historically involved offline techniques, such as culture-based methods and manual cell counting by optical fluorescence microscopy. These methods require long air sampling periods and significant post-collection labor, and they provide poor temporal resolution. In response to these shortcomings, a new generation of online, automated instruments for the measurement of PBAP, such as aerosol mass spectrometers (Tobias et al., 2005) and fluorescent particle spectrometers (Pan et al., 2003; Kaye et al., 2005), have recently been developed.

Measurements of single-particle fluorescence has been used for rapid detection of PBAP in the fields of atmospheric science (Pöschl et al., 2010), public health (Bhangar et al., 2015), and biological warfare research (Greenwood et al., 2009). Many biological compounds, including certain amino acids (e.g., tryptophan, tyrosine), metabolic small molecules (e.g., the reduced form of nicotinamide adenine dinucleotide, or NADH), and some proteins (e.g., green fluorescent protein), are intrinsically fluorescent (Chudakov et al., 2010). 
In single-particle fluorescence instruments, fluorescence in such compounds is induced using ultraviolet excitation, and the resulting fluorescence is detected either in relatively broad emission bands using filters or with high spectral resolution using a spectrometer. Fluorescent particle loadings are then used as a proxy for PBAP.

Despite the proliferation of single-particle fluorescence instruments (see Pan et al., 2003, and Kaye et al., 2005, for early prototype examples and the Ultraviolet Aerosol Particle Sizer [UV-APS; TSI, Inc.] and Wideband Integrated Bioaerosol Sensor, DMT, Inc., for commercially available examples), there is no standard method used to calibrate the magnitude of their fluorescence signals. Fluorescently dyed polystyrene latex spheres (FPSLs) are commonly used to assess detector performance, instrument alignment, and excitation pulse timing. FPSLs, however, have significant batchto-batch variability and suffer from poor shelf life; thus, they do not provide a repeatable, absolute calibration for fluorescence intensity. The lack of a standard limits our ability to compare observations made with different instruments, to track long-term instrument stability, and to assess the fundamental limit of detection (LOD) of the technique.

The amount of fluorescence produced by a single PBAP particle is potentially a useful metric for distinguishing and classifying particles detected in single-particle fluorescence instruments. The use of these fluorescence magnitudes, however, varies widely in the published literature. Several recent studies have employed a binary yes-no classification of fluorescence above a threshold (e.g., Gabey et al., 2010; Perring et al., 2015), essentially ignoring the fluorescence magnitude beyond the threshold. Fluorescence magnitudes have been used as input variables in automated particle-clustering analyses (Robinson et al., 2013; Crawford et al., 2015) and to manually sort sampled particles into groupings (Wright et al., 2014). Particles emitting so much fluorescence as to saturate detectors are sometimes excluded from analysis (e.g., Toprak and Schnaiter, 2013), and relatively weak fluorescence has been proposed as a possible discriminator of interfering nonbiological particles (Hill et al., 1999; Crawford et al., 2014, 2016; Yu et al., 2016). The utility of fluorescence magnitudes will increase greatly with the development of an absolute fluorescence calibration strategy applicable to any single-particle fluorescence measurement technology.

Here we present a reliable calibration strategy for fluorescence intensities measured by single-particle fluorescence instruments. Methods for solution preparation, particle generation, and data analysis are presented.

\section{Methods}

We evaluated the response of a Wideband Integrated Bioaerosol Sensor (Droplet Measurement Technologies, Boulder, CO, USA) to monodisperse aerosol particles containing a known mass of fluorescent material. These experi- ments were conducted using fluorophores emitting in one or more of each of the fluorescence detectors and for different detector gains in the WIBS-4A. The following criteria guided our selection of fluorescent material.

1. Fluorescent properties: fluorophores were chosen to match one or more of the excitation wavelengths (280, $370 \mathrm{~nm})$ and emission bands (310-400, 420-650 nm) of the WIBS-4A.

2. Stability: chemically inert fluorophores were chosen such that the signal from particles of a given size were constant over the course of a calibration.

3. Repeatability: the relationship (calibration curve) between fluorescence signal and fluorophore mass needed to be repeatable across multiple experiments with different batches of prepared solutions.

4. Availability and ease of preparation: all fluorophores used are inexpensive and easy to acquire. Importantly, each fluorophore chosen was soluble either in water or isopropanol for atomization.

5. Safety: the materials used are all relatively safe to handle and prepare, though proper personal protective equipment was worn and exposure to exhausted particles was avoided.

Tryptophan and quinine fulfilled these requirements. $\mathrm{NADH}$ and naphthalene were also tested, but each failed to meet one or more of the above requirements: the fluorescence signal from NADH was unstable, while the intensity of the signal from naphthalene was too low to be a suitable calibration material. The results from all materials tested are presented in Sect. 3. Below we present our detailed strategy for calibrating the fluorescence signals from the WIBS with these materials.

\subsection{Wideband Integrated Bioaerosol Sensor (WIBS-4A) operation}

We validated the procedure using a commercially available WIBS (model 4A), first described by Kaye et al. (2005) and later in significant detail by Gabey et al. (2010) and Perring et al. (2015). We will briefly describe its operating principles and the instrument settings used in this study.

The WIBS counts and sizes all incoming particles using elastic scattering from a continuous-wave laser $(635 \mathrm{~nm}$, $12 \mathrm{~mW}$ ). This scattering signal triggers the sequential flashing of two xenon lamps (5W L9455 modules, Hamamatsu Photonics K.K., Japan), one of which is filtered to emit light at 280 and the other at $370 \mathrm{~nm}$. Any resulting fluorescence is collected by two photomultiplier tubes (PMTs, H10720110, Hamamatsu Photonics K.K., Japan) filtered to detect only specific wave bands: the FL1 detector detects 310$400 \mathrm{~nm}$ emission, and the FL2 detector detects $420-650 \mathrm{~nm}$ 
emission, though the peak sensitivity for each detector is in the $350-450 \mathrm{~nm}$ range. A reference voltage input controls the gain on each PMT, which is changed manually with a variable potentiometer. We refer to this as the gain voltage throughout the rest of the paper. The FL2 detector also detects the scattering signal used for optical sizing. At reasonable particle sample rates, it can do so without interfering with the fluorescence measurement, since the scattering event and the two flash lamp pulses are separated in time. Three fluorescence signals are therefore recorded for a given particle: fluorescence between 310 and $400 \mathrm{~nm}$ following $280 \mathrm{~nm}$ excitation (referred to here as Channel A) and fluorescence between 420 and $650 \mathrm{~nm}$ with either 280 or $370 \mathrm{~nm}$ excitation (referred to as channels $\mathrm{B}$ and $\mathrm{C}$, respectively).

Both before and after the fluorescence calibration, the WIBS was run in forced trigger (FT) mode. In FT mode, the two xenon flash lamps are triggered automatically (as opposed to being triggered by the presence of a particle) at $\sim 2 \mathrm{~Hz}$ to assess the background light detected in each PMT in the absence of particles. The FT background in each detector is a function of the flash lamp intensity, the flash lamp alignment, the efficiency of the filter at rejecting the excitation wavelength, the detector gain setting, and fluorescence from any material deposited within the instrument cavity (Toprak and Schnaiter, 2013). In general WIBS data analysis, FT data are used to determine a signal threshold for each channel above which a particle is considered fluorescent. In ambient measurements, where a majority of particles are non-fluorescent, the fluorescence threshold can be assessed without taking the instrument offline to run in FT mode, as there generally exists a dominant population of nonfluorescent particles that have a distribution of fluorescence magnitudes identical to the background data collected in FT mode (Perring et al., 2015). Here, sample particles were fluorescent by design and we use $2-5 \mathrm{~min}$ of FT mode data to determine fluorescence thresholds for each channel. Gaussian functions were fit to FT signal peak intensity, and fluorescence thresholds were defined as three standard deviations above the center of the fitted Gaussian function $(\mathrm{FT}+3 \sigma)$.

The timing of the firing of each flash lamp was set using the optimization function in the WIBS acquisition software while sampling monodisperse fluorescent particles. FPSLs were typically used for this process, though the sizeselected calibration particles presented here work as well. The timing optimization program scans through a wide range of delay times for the lamps for a given fluorescence channel following triggering (detecting the scattered light pulse). The software simultaneously averages fluorescence signals for each delay time, and the delay time corresponding to the maximum average fluorescence signal determines the optimal flash lamp timing. Flash lamp timing was periodically determined for each fluorescence channel but did not vary over the course of these measurements.

All experiments presented here use a WIBS sample flow rate of $0.3 \mathrm{~L} \mathrm{~min}^{-1}$, and a sheath flow rate of $2.1 \mathrm{~L} \mathrm{~min}^{-1}$,

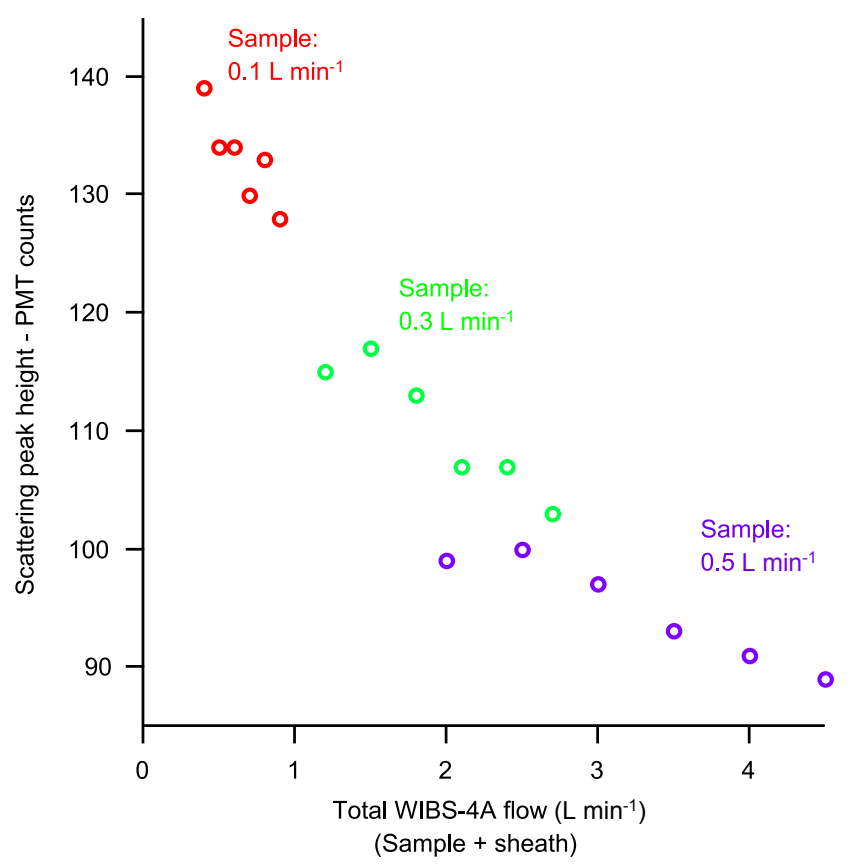

Figure 1. Scattering signal dependence on total WIBS flow rate (sample + sheath) in $\mathrm{L} \mathrm{min}^{-1}$. Data collected at a given sample flow rate are colored similarly. All data shown are for $1100 \mathrm{~nm}$ dioctyl sebacate particles.

close to typical factory settings. Laboratory tests reveal an inverse relationship between optical scattering peak height and particle velocity, as shown in Fig. 1. This relationship likely is attributable to insufficient signal processing speed to fully resolve the scattering peak magnitude, a problem exacerbated at higher particle velocities. While the curve shown in Fig. 1 is steepest at the lowest combined sheath and sample flows, and thus would be more susceptible to sizing errors due to flow fluctuations, the mass flow controllers (MFCs) used in the WIBS have stated accuracies with $1 \%$ of their reading. Thus, we don't expect large sizing errors as a result of flow variability. Conversely, the increasing inability of the WIBS to resolve the true peak height at higher flow rates will lead to large uncertainties in optical sizing. While we recommend running the instrument at low combined flow rates, experimental constraints may dictate the need for other flow configurations. Regardless, what is most important is that instrument response, as well as flash lamp timing, is dependent on the combined flow rate. Calibrations should be performed for any flow configuration used.

\subsection{Particle generation and sampling}

The experimental setup used for WIBS calibration consisted of three general components: particle generation, particle conditioning, and measurement. This is shown schematically in Fig. 2. 
(a)

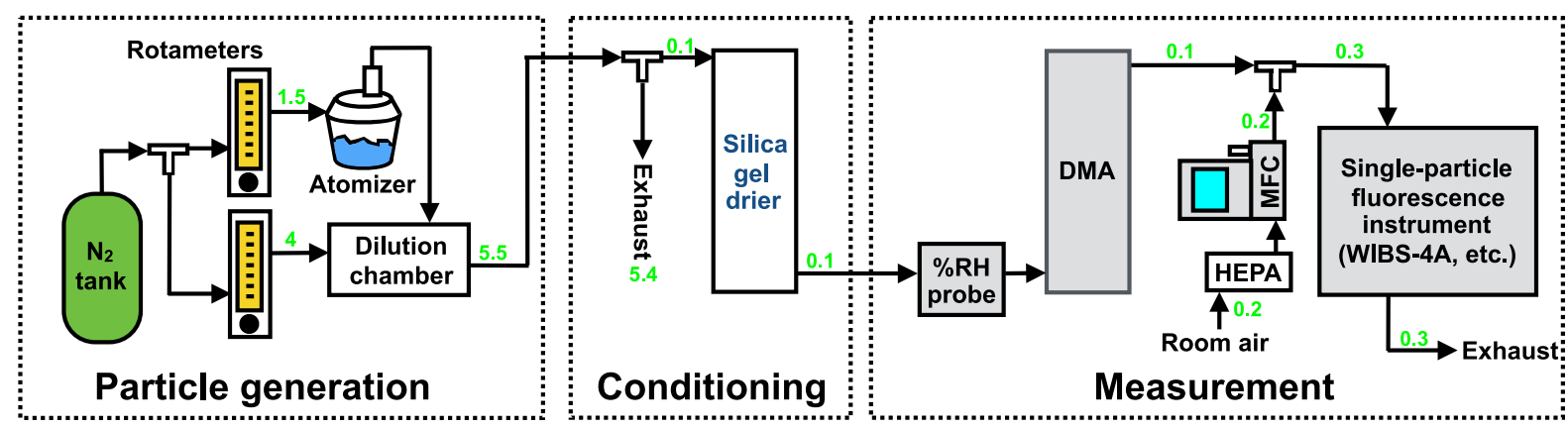

(b)

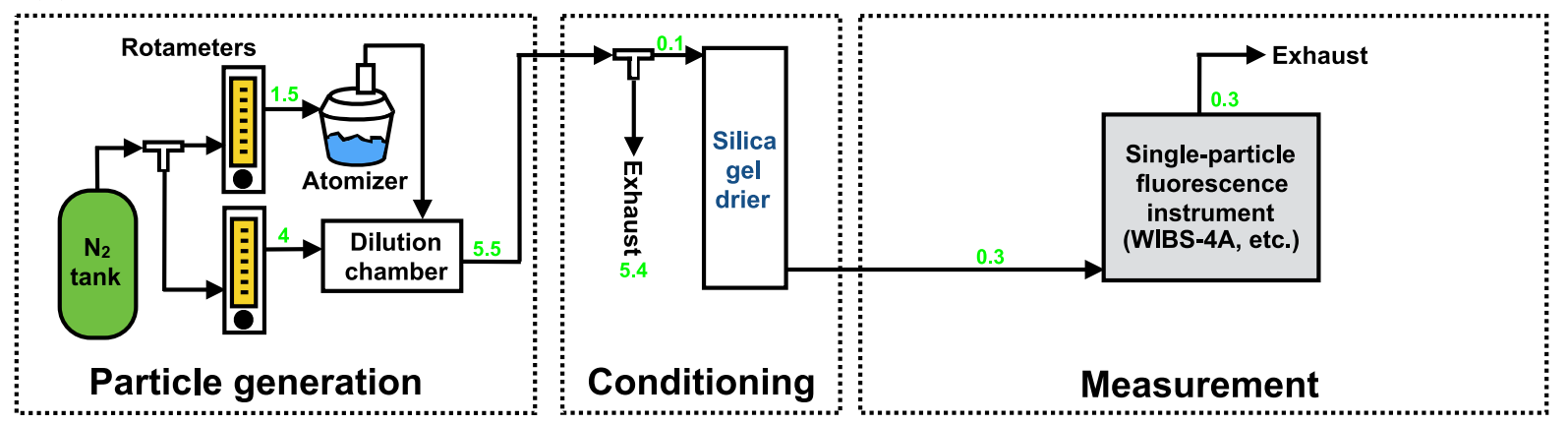

Figure 2. Experimental setup for fluorescent particle calibrations. Green numbers indicate flow rates in L $\mathrm{min}^{-1}$. (a) Size-selected calibration experiments. (b) Polydisperse calibration experiments.

Fluorescent particles were generated by nebulizing a solution containing a fluorophore dissolved in either isopropanol (99.9\% purity, HPLC-grade, Pharmco) or deionized water, depending on the solubility of the fluorescent material. Additional non-fluorescent material was added to the nebulized solution, as needed, to adjust the per-particle mass of fluorophore. The desired range of fluorophore masses was determined empirically based on typical factory gain settings and previous observations of fluorescence magnitudes of known biological materials. For example, pure quinine ( $>98 \%$, Sigma-Aldrich) particles within the size range of the WIBS $(>0.8 \mu \mathrm{m})$ exhibited fluorescence intensities that were within the dynamic range of the detector at typical gain settings. For tryptophan (L-tryptophan, $>98 \%$, Sigma-Aldrich) on the other hand, pure particles saturated the detector at typical gain settings and produced much higher fluorescence signals than biological materials of comparable size. That the instrument is more sensitive to tryptophan than quinine on a mass basis is likely due to the peak sensitivity of the WIBS PMTs overlapping significantly with the tryptophan emission spectrum and less so with that of quinine (Pant et al., 1990; Goldberg et al., 2012). Therefore, tryptophan-based calibration particles were an internal mixture of tryptophan and ammonium sulfate "filler". Ammonium sulfate $(\geq 99 \%$, Sigma-Aldrich) was chosen because it is very soluble in water and has previously been shown not to fluoresce in the
WIBS (Toprak and Schnaiter, 2013). In this case, the nebulized solution was prepared by mixing appropriate volumes of each stock solution (e.g., tryptophan in water and ammonium sulfate in water), with care taken to ensure the solution was well mixed before nebulization. The composition of the particles is assumed to match that of the non-volatile components of the bulk solution. A full list of the gravimetric solutions used is presented in Table 1.

A nitrogen tank or high-efficiency particulate air (HEPA)filtered room air was used to supply pressure to the nebulizer and a sealed dilution volume with inlet and outlet ports. The flows to the nebulizer and the dilution chamber were both controlled with rotameters tuned to provide adequate flow to the nebulizer while sufficiently diluting the output aerosol to maintain a manageable particle sample rate in the WIBS (limited by the recharge time of the flash lamps). The minimum flow rate required for the medical nebulizers used here (B\&F AeroMist Nebulizer, Allied Healthcare Products, Inc.; St. Louis, MO, USA) was determined to be $\sim 1 \mathrm{~L} \mathrm{~min}^{-1}$, though this depended slightly on the solution. Typical dilution flow rates were $3-5 \mathrm{~L} \mathrm{~min}^{-1}$, yielding a total output flow of 4-6 $\mathrm{L} \mathrm{min}-1$. A bypassing port (a simple T-union with one end open to ambient) was installed downstream of the dilution chamber and upstream of a diffusion drier containing Drierite (anhydrous $\mathrm{CaSO}_{4}$ ), which reduced the $\mathrm{RH}$ of the aerosol stream to $<1 \%$. The output from the drier fed into a 
Table 1. List of gravimetric solutions used for calibration experiments.

\begin{tabular}{lllrrr}
\hline Expt \# & $\begin{array}{l}\text { Mass \% } \\
\text { FL solution }\end{array}$ & $\begin{array}{l}\text { Mass \% } \\
\text { non-FL solution }\end{array}$ & $\begin{array}{r}\text { FL solution } \\
{[\mathrm{mL}]}\end{array}$ & $\begin{array}{r}\text { non-FL solution } \\
{[\mathrm{mL}]}\end{array}$ & $\begin{array}{r}\text { Mass \% fluorophore } \\
\text { (in dry particles) }\end{array}$ \\
\hline 1 & 0.648 Trypt. (Iso.) & $0.090 \mathrm{AS}\left(\mathrm{H}_{2} \mathrm{O}\right)$ & 3 & 9 & 2.35 \\
2 & 0.832 Trypt. (Iso.) & $0.090 \mathrm{AS}\left(\mathrm{H}_{2} \mathrm{O}\right)$ & 3 & 9 & 2.99 \\
3 & 0.648 Trypt. (Iso.) & $0.090 \mathrm{AS}\left(\mathrm{H}_{2} \mathrm{O}\right)$ & 3 & 9 & 2.35 \\
4 & 0.648 Trypt. (Iso.) & $0.090 \mathrm{AS}\left(\mathrm{H}_{2} \mathrm{O}\right)$ & 3 & 9 & 2.35 \\
5 & 0.959 Quin. (Iso.) & - & 15 & - & 100.00 \\
6 & 0.778 Quin. (Iso.) & - & 15 & - & 100.00 \\
7 & 0.514 Quin. (Iso.) & - & 15 & - & 100.00 \\
8 & 1.341 Quin. (Iso.) & - & 15 & - & 100.00 \\
9 & 0.535 Quin. (Iso.) & - & 15 & - & 100.00 \\
10 & 0.514 Quin. (Iso.) & - & 15 & - & 100.00 \\
11 & 6.332 Naph. (Iso.) & - & 15 & - & 100.00 \\
12 & 0.050 NADH (0.01 M NaOH) & - & & 57.29 \\
\hline
\end{tabular}

differential mobility analyzer (DMA; custom-built at NOAA, Boulder, CO USA). It should be noted that the custom-built DMA has a longer column than some commercially available versions (e.g., TSI 3081), and so it is more easily able to select larger size particles.

The DMA was used to select a narrow size range of the incoming polydisperse aerosol for sampling by the WIBS. We calculated the per-particle mass of fluorophore based on the selected particle mobility diameter and the mass fraction of nebulized solution assuming dry spherical particles. This mass provides the basis for our calibration scheme, as it associates the fluorescence signals from the WIBS with an absolute fluorescent mass. This experimental setup is shown in Fig. 2a.

The flow rate of the aerosol stream through the diffusion drier and the DMA is controlled by the WIBS flow rate $\left(0.3 \mathrm{~L} \mathrm{~min}^{-1}\right)$ and a mass flow controller downstream of the DMA. A sample flow through the DMA of $0.1 \mathrm{~L} \mathrm{~min}^{-1}$ was optimal to select particles in the size range of interest $(650 \mathrm{~nm}-3 \mu \mathrm{m})$. We used a DMA sheath flow of $1 \mathrm{~L} \mathrm{~min}^{-1}$ (10: 1 sheath to sample ratio). A makeup flow of $0.2 \mathrm{~L} \mathrm{~min}^{-1}$ of HEPA-filtered lab air was controlled by the MFC after the DMA. It should be noted that this makeup flow was not dried. This $2: 1$ dilution from the makeup flow did not result in detectable evaporation or water uptake of the size-selected particles, as determined by comparing measured particle sizes with and without this dilution, nor did it affect their fluorescence signals. However, drying this makeup flow would remove any potential for water uptake and could potentially be important in more humid environments. An RH probe (INTERCAP HMO60, Vaisala; Helsinki, Finland) installed inline between the drier and the DMA showed that the measured humidity of the stream was between $\mathrm{RH}=1$ and $2 \%$, which indicated that particles were thoroughly dried prior to sizing. We estimate the residence time in the drier to be roughly $1.7 \mathrm{~min}$.
In this configuration, the WIBS-4A sampled a stream of monodisperse aerosol particles with a known mass of fluorescent molecules, and the resulting fluorescence signal magnitudes were analyzed. We performed these experiments at several gain voltage settings and reported the full results in Sect. 3. The experiment was conducted within a fume hood to contain all exhaust particles. In the absence of a fume hood, particulate filters on the exhaust lines are recommended to minimize any potential exposure to calibration particles.

\subsection{Calibration procedure}

The starting point for most calibrations is the preparation of the gravimetric fluorescence standard solution. The solutions used in these calibrations are listed in Table 1 . We have verified that the signals from quinine and tryptophan do not significantly degrade over the course of 2 days under the preparation and storage procedures used here. We have not assessed the stability of these solutions for longer terms and recommend solution preparation for semi-immediate use. Following the solutions preparation, $12-15 \mathrm{~mL}$ was transferred to a clean medical nebulizer. This volume of solution was sufficient to last for $1.5 \mathrm{~h}$, the maximum duration of a typical calibration experiment. Unused solutions were stored in a dark refrigerator.

Prior to sampling fluorescent particles, the WIBS was run in FT mode for $\sim 5 \mathrm{~min}$. Following FT data collection, pressurized nitrogen was provided to the system, starting fluorescent particle generation as described above. The rotameter controlling the dilution flow upstream of the DMA was adjusted to provide a particle count rate in the WIBS of $<100$ counts $\mathrm{s}^{-1}$, below the maximum duty cycle of the flash lamps ( 125 counts $\mathrm{s}^{-1}$ ), which allows for detection of all particles. Typical particle concentrations in these experiments produced 10-100 counts $\mathrm{s}^{-1}$, depending on solute concentration in the nebulizer, selected mobility diameter, and dilution flow. For each selected mobility diameter, approximately 


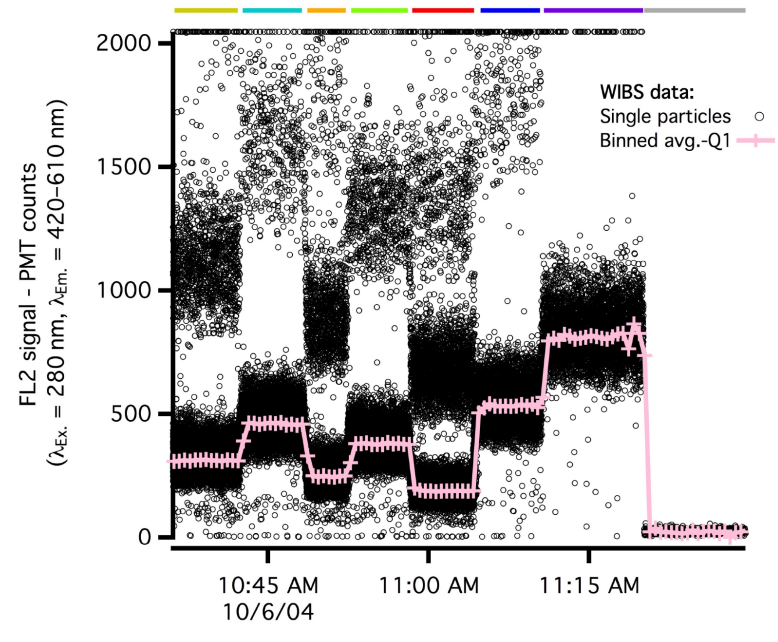

(a)

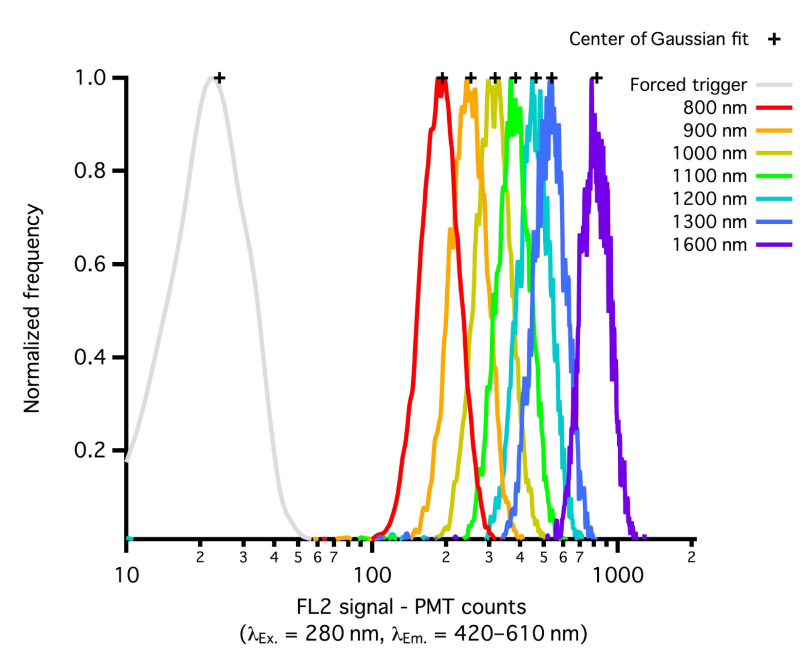

(b)

Figure 3. Example time series data for calibration experiments illustrated by showing (a) FL2 WIBS data points for different sizes of mobility-selected particles and (b) histograms of singly charged fluorescent calibration particles. Colored bands at the top of (a) correspond to the selected particle sizes shown in the legend of (b). This example illustrates the typical timeline of a calibration experiment for quinine and how we analyze the data to construct calibration curves (shown in Fig. 4). Only singly charged particles were used to construct the calibration curves.

5 min of data were collected, providing enough particles to build high-fidelity histograms for determination of the central value and variability of fluorescence using Gaussian fits. After a sufficient amount of data was collected, the voltage on the DMA was changed to select for another particle size, and this process was repeated until all data from all target sizes was collected. Data shown in the example experiment depicted in Fig. 3 span the particle size range of $650 \mathrm{~nm}$ to $2.5 \mu \mathrm{m}$. Following data collection from size-selected fluorescent particles, FT mode data was collected again for $\sim 5 \mathrm{~min}$.

\subsection{Data analysis}

A time series of single-particle data from a typical calibration of the FL2 detector using pure quinine particles is shown in Fig. 3a. Black circles show individual fluorescence values, and the pink line shows the $30 \mathrm{~s}$ average fluorescence intensity for the singly charged ("Q1") population. Mobility diameters selected by the DMA are labeled with colored bands above the plot. Data are discarded during transitions from one DMA voltage to another (typically requiring $\sim 30$ s), indicated by small gaps between the colored bands.

We construct fluorescence intensity histograms for each size (shown in Fig. 3b) and fit a Gaussian function to each singly charged mode, the center of which represents the modal fluorescence intensity for that size. In Fig. 3b, each histogram is normalized to its maximum value and colored according to the colored bands in Fig. 3a. We calculate the mass of fluorescent material for a given size by assuming a spherical particle shape and complete removal of solvent. In the case of the mixed tryptophan-ammonium sulfate particles, we assume that the mass fraction in the dry particles is the same as in the atomizer solution. Fluorescence intensities as a function of fluorophore mass are then used to construct the calibration curves shown in Fig. 4, which are discussed further in Sect. 3.

\subsection{Polydisperse fluorescent aerosol calibrations}

We also performed fluorescence calibrations using a polydisperse stream of fluorescent aerosol particles. Fluorescent particles were prepared in a similar manner as above, with the exception that no DMA was used to size-select from the aerosol stream. This modified experimental setup is shown in Fig. 2b. Typical flow rates are shown in green in Fig. 2b, but sometimes much higher dilution flow rates were used to ensure a particle count rate in the WIBS below $125 \mathrm{~s}^{-1}$.

Data were analyzed similarly to above, except WIBS scattering signals were used to provide particle size. A smoothed Mie curve (so as to be monotonically increasing) based on our instrument's geometry was used as a sizing calibration for the scattering signals. This sizing calibration curve was derived for each aerosol type used (quinine and ammonium sulfate-tryptophan) and for each gain setting based on sizeselected data. Each sizing calibration curve was then applied to scattering signals from the polydisperse aerosol data to provide particle diameter, which was converted to mass assuming complete drying and spherical particle shape. It should be noted that the application of a monotonically increasing size calibration curve does not necessarily capture 


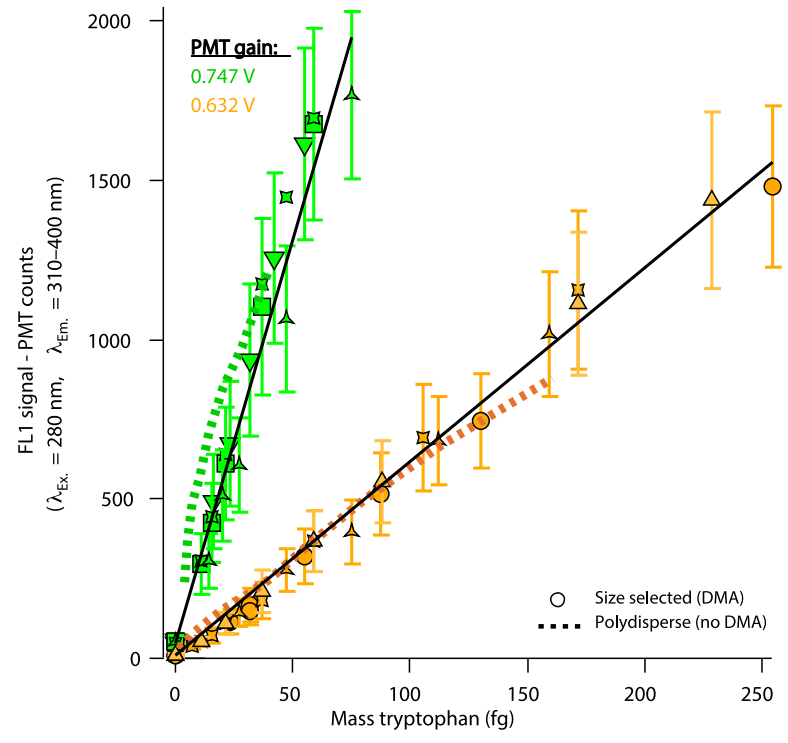

(a)

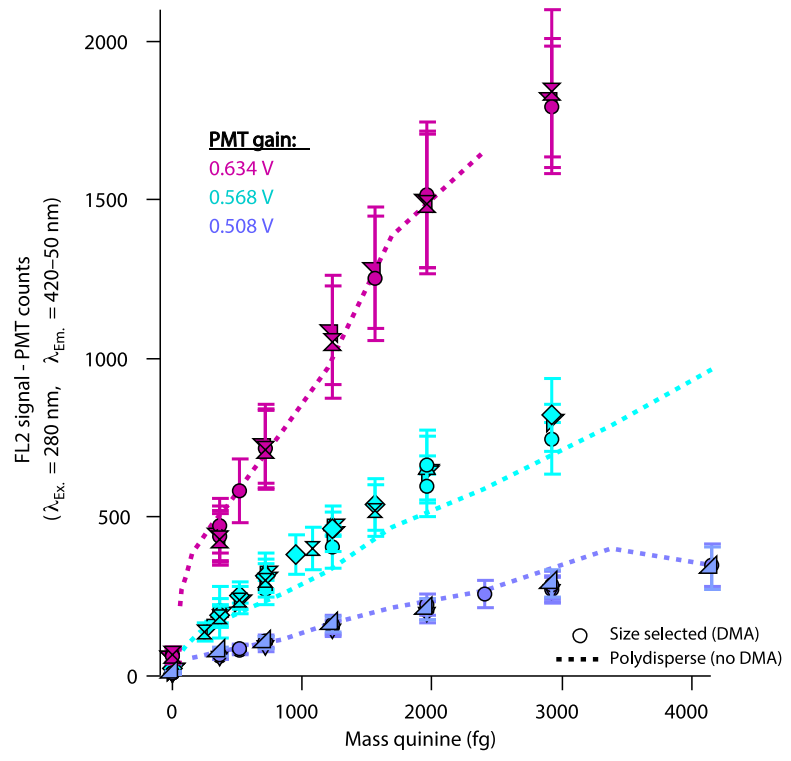

(b)

Figure 4. Fluorescence calibration curves for the FL1 (a) and FL2 (b) detectors for different PMT detector gains. The FL1 and FL2 detectors are calibrated with mixed tryptophan-ammonium sulfate and pure quinine particles, respectively. Each data point represents the central value of a Gaussian function fit to fluorescence signals at a given particle mass, while the error bars are the standard deviation in fluorescence signals for that mass. Each symbol shape refers to data from an individual calibration experiment to show repeatability. Dotted lines show results from polydisperse, fluorescent aerosol calibration experiments. Black lines in (a) are linear fits to the monodisperse data.

all of the Mie scattering behavior. Thus, due to sizing errors, the polydisperse calibration slopes are expected to be less robust than those generated using a DMA. Individual fluorescence signals were binned by size, and Gaussian functions were fit to determine the modal fluorescence signal for a given bin. Only data from bins with $>500$ measured particles are displayed in Fig. 4.

\subsection{Xenon flash lamp intensity tests}

The relationship between fluorescence intensity and excitation power in fluorescence measurements can be complex. For instance, with too-high excitation power there can be saturation effects where fluorescent molecules are photobleached (Faris et al., 1997) or their excited states are depopulated through stimulated emission (Georges et al., 1996). With too-low excitation energy, fluorophores may not exhibit fluorescence at all (Kaye et al., 2005). Thus, the power of the excitation radiation can potentially have a large impact on the magnitude of fluorescence light measured.

The WIBS provides a measurement of the relative power output of each xenon flash lamp pulse with a fiber-optic sensor, which was designed to provide a measure of flash power over the lifetime of the lamp. These sensors, consisting of a silicon PIN photodiode, which are placed near the arc lamp in the lens tube, provide a current that scales relative to the amount of light measured from each flash. However, they only provide a relative measure of light, and not an absolute measure of the excitation energy experienced by each particle detected by the WIBS. Moreover, this measurement is highly sensitive to the placement of the fiber-optic sensor within the lens tube of the flash lamp and may not provide a repeatable measurement when the fiber-optic sensor is moved, either through instrument vibrations or when removing the flash lamp module for maintenance. In order to assess the response of our calibration particles to changes in lamp power, we performed fluorescence calibration tests using neutral density filters with varying optical densities inserted within the lens tube of the flash lamps. Additionally, we performed our calibrations both with the original flash lamp, which has been installed in our instrument since its purchase ( $\sim 3$ years ago), and a new flash lamp, in order to assess any possible degradation in power due to prolonged use.

\section{Results and Discussion}

\subsection{Calibration results}

Compiled results from the tryptophan and quinine calibrations are shown in Fig. 4a and b, respectively, constructed as described above from single-particle fluorescence intensities measured for various fluorophore masses. Different symbols 


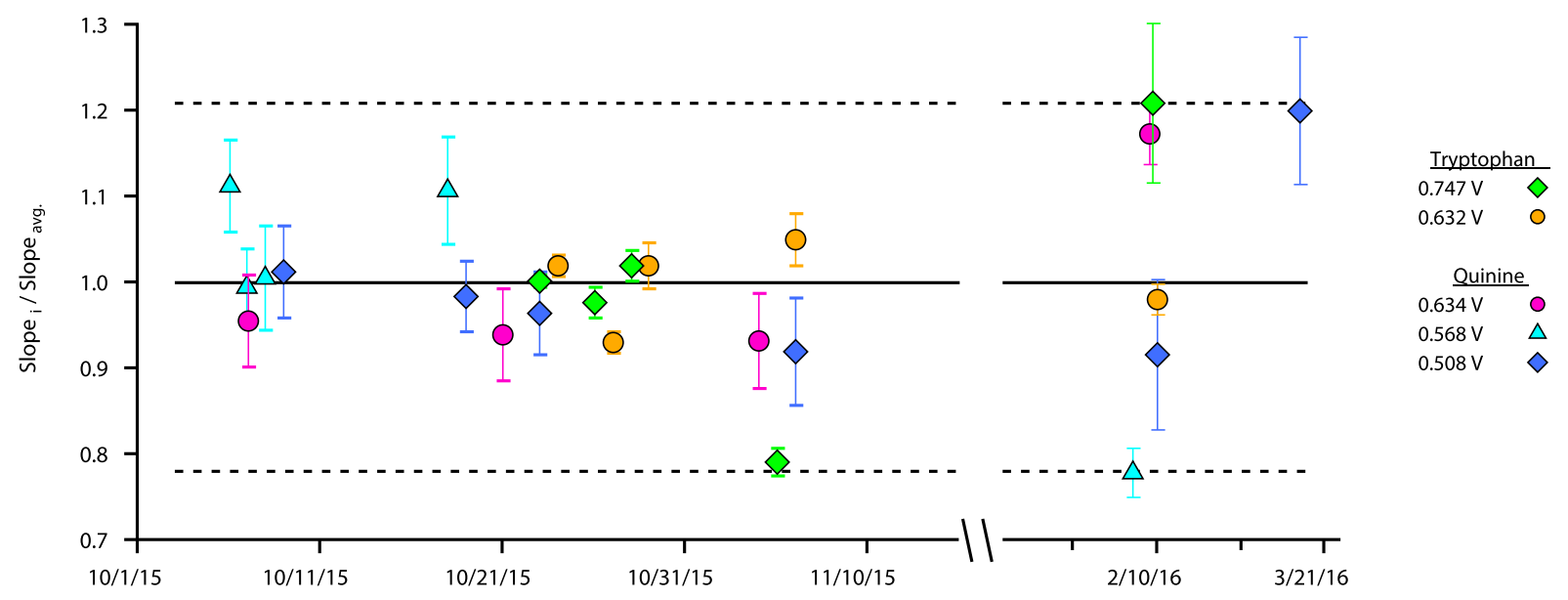

Figure 5. Normalized calibration curve slopes shown for the duration of the study for both calibration materials at each gain setting tested. The $y$ axis is the slope of an individual experiment normalized by the average slope of all experiments of a given calibrant and gain. Error bars represent \pm the standard deviation of the slope fit to each experiment. Some of the data points have been shifted by a \pm 1 day to avoid symbol overlap for visual purposes. The range of all calibration experiments is $\pm 22 \%$.

correspond to individual calibration experiments, and the error bars represent the width of the Gaussian fits to observed fluorescence intensities for a given mass. For both particle types we also tested different PMT detector gain voltages. All gravimetric standard solutions were prepared within 2 days of a given experiment. We collected FT mode data prior to each experiment, which is also shown on these graphs. The linear fits shown in Fig. 4a are constrained such that the $y$ intercept is equal to the average fluorescence signal from FT mode (second column in Tables 2 and 3).

The detector gain clearly has a significant impact on the detector response for a given mass of fluorescent material. For instance, for the two FL1 gain settings shown in Fig. 4a, the high gain $(0.747 \mathrm{~V})$ slope $\left(25.0 \pm 1.7\right.$ counts $\mathrm{fg}^{-1}$ tryptophan) is $\sim 4$ times higher than for the low gain $(0.632 \mathrm{~V})$

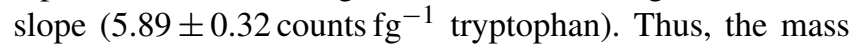
of tryptophan that saturates the FL1 detector at the high gain setting is $\sim 4$ times lower than the low gain setting. As mentioned earlier, two WIBS clustering studies (Robinson et al., 2013; Crawford et al., 2015) excluded saturating particles from their analysis, and so understanding the range of measurable fluorophore mass is potentially important.

In Fig. 4a we show an average linear fit to all data for mixed ammonium sulfate-tryptophan particles, and the slope of the reported calibration curves in Table 2 is the average slope across all of the individual experiments. Slopes for individual calibration experiments, for a given FL channel and gain, were within $22 \%$ of the average slope, and between 4 and 6 experiments were performed at each gain setting. We take the linearity of the calibration curve shown for tryptophan in Fig. 4a as evidence that there is not significant shielding or quenching of fluorescence over the fluorophore concentrations and particle sizes studied here for the mixed tryptophan-ammonium sulfate particles. While there is noise in the data points shown in Fig. 4, there are no systematic biases or individual experiments that are outliers, as the data from all experiments are distributed around the linear fit. The stability of these gain curves was assessed over a period of 4 months. A time series showing the stability of the calibration slopes is shown in Fig. 5.

Quinine, on the other hand, produced a nonlinear relationship between measured fluorescence and particle mass. This is shown for an example calibration experiment in Fig. 6a. The largest particles in this study produced somewhat less fluorescence signal per mass than the smaller particles, likely indicating that quinine in the center of the particle is not contributing to the observed fluorescence. Figure $6 \mathrm{~b}$ shows the relationship between fluorescence and participating quinine mass, shown for an example penetration depth of $90 \mathrm{~nm}$. This renders a linear relationship between quinine mass and fluorescence intensity. We assume that quinine mass in this outer shell fully participates in fluorescence, while mass at the center of the particle does not participate at all. A penetration depth of $90 \mathrm{~nm}$ resulted in the best linear fit for the instrument used in these experiments, as determined by optimizing $R^{2}$ for fits over a range of penetration depths used for this calculation. A full analysis of factors affecting the penetration depth (such as lamp intensity or instrument alignment) requires further investigation, and the appropriate depth may vary from instrument to instrument. Nevertheless, the quinine calibration gives a repeatable response over time for a given instrument and provides a basis for comparing fluorescence intensities in the FL2 detector observed by different instruments.

The variability in fluorescence signals in these calibration experiments are represented by error bars in Fig. 4. These error bars are the width of the Gaussian fits to the fluorescence intensities at a given particle size. They represent the 
Table 2. Calibration responses and limits of detection for tryptophan for different gain voltage settings.

\begin{tabular}{lrrrrr}
\hline $\begin{array}{l}\text { Gain } \\
(\mathrm{V})\end{array}$ & $\begin{array}{r}\mathrm{FT} \\
\text { (counts) }\end{array}$ & $\begin{array}{r}\mathrm{FT}+3 \sigma \\
\text { (counts) }\end{array}$ & $\begin{array}{r}\text { Slope } \pm \text { SD } \\
\left(\text { counts fg }^{-1}\right)\end{array}$ & $\begin{array}{r}\text { Lower LOD } \\
\text { (fg tryptophan) }\end{array}$ & $\begin{array}{r}\text { Upper LOD } \\
\text { (fg tryptophan) }\end{array}$ \\
\hline 0.747 & 50.0 & 105 & $25.0 \pm 1.7$ & 2.17 & 79.9 \\
0.632 & 11.1 & 24.3 & $5.89 \pm 0.32$ & 2.18 & 335 \\
\hline
\end{tabular}
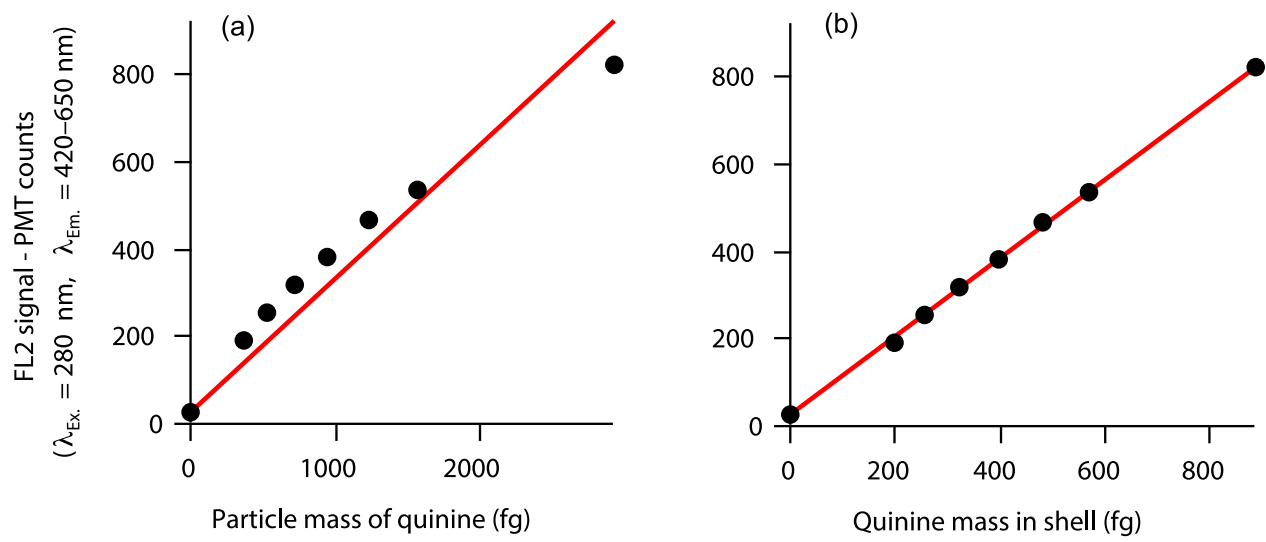

Figure 6. Relationship between fluorescence measurements and quinine mass for (a) particles and (b) shells of $90 \mathrm{~nm}$ thickness, for an illustrative calibration experiment where the FL2 detector is at the medium gain setting. Red lines are linear fits constrained to pass through the forced trigger data, shown by the vertical intercept at quinine mass equal to zero. The plot shown in panel (a) illustrates that the relationship between measured fluorescence and quinine mass is nonlinear across the mass range measured. The relationship between measured fluorescence and mass becomes linear when we assume a penetration depth where only quinine in the outer shell contributes to the observed fluorescence, shown in panel (b).

Table 3. Calibration responses and limits of detection for quinine for different gain voltage settings.

\begin{tabular}{lrrrr}
\hline $\begin{array}{l}\text { Gain } \\
\text { (V) }\end{array}$ & $\begin{array}{r}\text { FT } \\
\text { (counts) }\end{array}$ & $\begin{array}{r}\text { FT }+3 \sigma \\
\text { (counts) }\end{array}$ & $\begin{array}{r}\text { Lower LOD } \\
\text { (fg quinine) }\end{array}$ & $\begin{array}{r}\text { Upper LOD } \\
\text { (fg quinine) }\end{array}$ \\
\hline 0.634 & 64.8 & 132 & 98.4 & $2.90 \mathrm{e} 3$ \\
0.568 & 23.7 & 49.7 & 83.9 & $6.55 \mathrm{e} 3$ \\
0.508 & 10.1 & 21.5 & 122 & $2.17 \mathrm{e} 4$ \\
\hline
\end{tabular}

standard deviation of single-particle fluorescence values observed for a given mobility diameter selected by the DMA and are typically $\pm 20 \%$. Because we are atomizing a wellmixed solution, we assume that all particles generated are uniform in composition, and so the variability in fluorescence can be attributed either to inherent noise in the WIBS, the transfer function of the DMA, or some combination of both. The variability in fluorescence intensity is equivalent to a $\sim 20 \%$ variability in fluorophore mass, which is roughly the width of the DMA transfer function on a mass basis. Thus, these error bars do not depict the fundamental precision of the WIBS itself, but rather the precision of this calibration technique using a DMA to select a narrow particle size range.

Also shown in Fig. 4 are results from the polydisperse fluorescent aerosol calibrations (dotted colored lines). The flu- orescence signal determined from the polydisperse aerosol data is within the error bars of the size-selected data points for almost all masses for both tryptophan and quinine. These data indicate that a polydisperse stream of fluorescent particles can provide an important qualitative (if not quantitative) check for fluorescence detector performance for the WIBS4A. The ability to roughly determine a fluorescence calibration curve that does not necessitate the use of a DMA makes this test a quicker, cheaper, and more field-deployable option for WIBS fluorescence calibration. However, due to the variability in optical size determination in the WIBS, this polydisperse fluorescence calibration method is not suggested as a substitute to using a DMA, but rather a supplemental technique that can be more easily and regularly applied.

\subsection{Effect of mass fraction tryptophan on fluorescence in mixed particles}

For mixed particles containing tryptophan and ammonium sulfate, there was a dependence on the mass fraction of tryptophan in the atomizer solution for the measured fluorescence in the FL1 detector. Figure 7 shows calibration curves for particles containing different calculated mass fractions of tryptophan, the slopes of which increase with increasing mass fraction. For the range of mass fractions explored here (1-4\% tryptophan in the mixed particles), we see an increase 


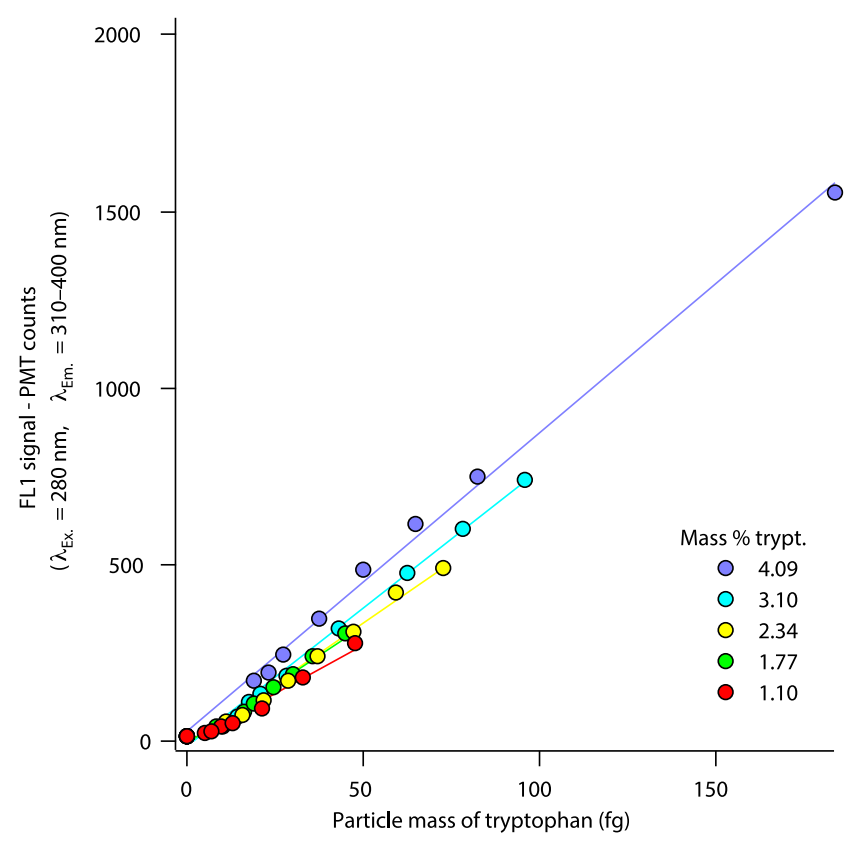

Figure 7. Fluorescence response to size-selected mixed particles containing tryptophan and ammonium sulfate at different mass ratios. These calibration curves were taken at a lower gain setting than either of the curves in Fig. 4a and thus should not be directly compared.

of $50 \%$ in the calibration slope for the particles with the most tryptophan (mass fraction $=0.0409$, purple) compared to the particles with the least tryptophan (mass fraction $=0.0110$, red). The mass fractions displayed in the legend correspond to the calculated dry mass fractions in the particles and are comparable to the last column in Table 1.

We were not able to determine the mechanism that explains this dependence on tryptophan mass fraction. Nonetheless, the trend was repeatable across many experiments. Additionally, we found similar results for mixed particles of tryptophan and dioctyl sebacate, which implies that the effect can not be attributed specifically to the presence of ammonium sulfate. Therefore, our calibration for the FL1 detector using the mixed particles is only "absolute" for the narrow concentration range (2.35-2.99\% tryptophan) for the specific chemicals used here. However, given the repeatability of our experiments, other users should be able to replicate our results using solutions prepared at the same nominal mass fraction. It is worth noting that while the difference in measured fluorescence intensity between the different solutions was significant, as determined by comparing the slopes of the fitted calibration curves, this difference is far less than the difference between the average slopes for the two gain settings tested and displayed in Fig. 4.

Quinine, on the other hand, does not suffer this limitation as a calibrant, as pure quinine particles (as opposed to mixed particles) exhibit fluorescence intensities within the range of

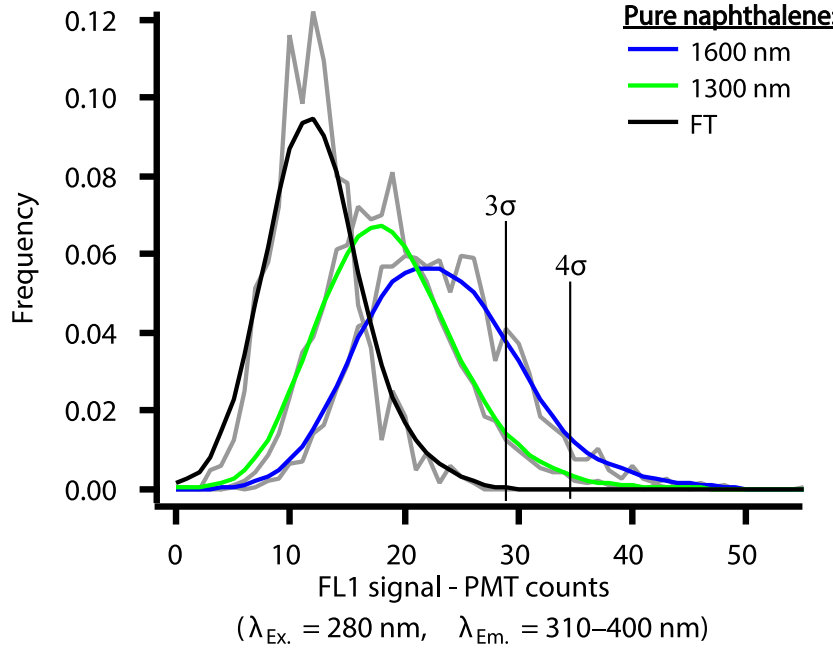

Figure 8. Fluorescence intensity histograms from the FL1 detector of size-selected naphthalene particles and forced trigger (FT) background for a gain setting of $0.632 \mathrm{~V}$. Gray lines are the raw data, while colored lines are smoothed for visualization purposes for each size and for FT. Only the tails of the naphthalene distributions are above the fluorescence thresholds ( $3 \sigma$ and $4 \sigma$ thresholds for this gain setting are indicated by vertical black lines).

values needed to calibrate these detectors. Thus, there may exist a more suitable calibrant than tryptophan for the FL1 detector in the WIBS. This calibrant would exhibit fluorescence intensity within the desired range for the FL1 detectors for pure particles of sizes in the WIBS detection range. This substance could be sampled in the form of pure particles, thus not requiring "dilution" on a per-particle basis, as in this case with tryptophan. Of course, an alternate calibration material for FL1 would still need to meet the requirements listed in Sect. 2.

\subsection{Failed calibrants: NADH and naphthalene}

We performed calibrations for several other fluorescent materials and found them to be inadequate as calibration standards for instruments like the WIBS. For example, naphthalene particles, formed by nebulizing a solution of naphthalene (>99\%, Sigma-Aldrich) dissolved in isopropanol (see Table 1), registered signal in our FL1 detector at a gain setting of $0.632 \mathrm{~V}$ but were at the threshold of detection. As shown in Fig. 8, only 22.5 or $7.5 \%$ of $1600 \mathrm{~nm}$ naphthalene particles registered above the FL1 threshold at a gain setting of $0.747 \mathrm{~V}$, for FT $+3 \sigma$ ( 29 counts) or FT $+4 \sigma$ (35 counts) thresholds, respectively. We experimented with more concentrated naphthalene solutions (to shift the polydisperse output from the nebulizer to larger sizes, making available more particles to be selected at higher DMA voltages), but these concentrations were so high that they clogged our nebulizers. However, it is worth noting that pure $1600 \mathrm{~nm}$ naphthalene particles $(\sim 2500 \mathrm{fg})$ had very low fluorescence. 


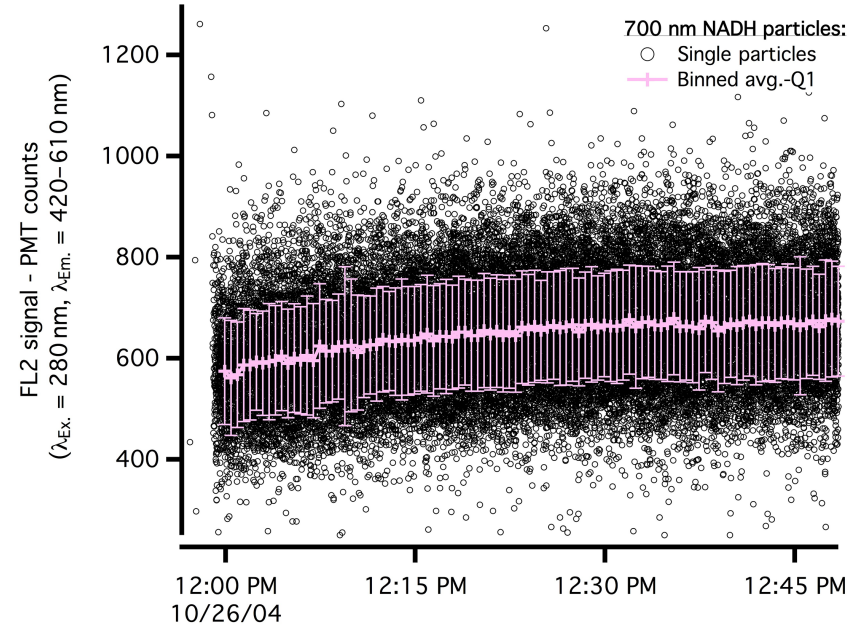

Figure 9. Time series from an example NADH calibration experiment, illustrating the lack of stability in the NADH fluorescence signal over $\sim 1$ h timescales. Signal from the FL2 detector is plotted on the $y$ axis for individual particles (black data points) and 1 min averages (pink line with error bars representing the standard deviation).

Reduced nicotinamide adenine dinucleotide (NADH) was also explored as a calibrant for the FL2 detector (MP Biomedicals, LLC). It showed strong signal in both channels imaged by the FL2 detector (channels B and C); however signals from a given particle diameter showed systematic drift over long ( hour) timescales. As shown in Fig. 9, the average signal observed in Channel B for $700 \mathrm{~nm} \mathrm{NADH}$ particles (1 min averages) steadily increased over the course of $1 \mathrm{~h}$, from 550 to 675 counts. These data are for an FL2 detector gain setting of $0.568 \mathrm{~V}$. In other experiments (not shown), the average signal from NADH particles would oscillate on similar timescales. For comparison, neither quinine nor tryptophan exhibited this drift in fluorescence. This behavior is presumably due to ongoing chemistry leading to variable conversion in the atomizer volume between different oxidation states of NADH, only one of which is fluorescent. While NADH-fluorescence is widely used to study enzymatic reactions (Lawkowicz et al., 1992), it is known to be highly unstable in solution (Rover, 1998) and the oxidized form (NAD+) is not fluorescent (Rost, 1992). So while NADH is an appealing potential calibrant for the WIBS, since it is a common metabolic molecule that exhibits fluorescence in the FL2 detector following both excitation wavelengths, stability issues prevented us from fully assessing it as we have done for quinine and tryptophan. However, we can say that the fluorescence signal from NADH and quinine particles is on the same order of magnitude: NADH as we have prepared it here is roughly twice as fluorescent as quinine particles of the same fluorophore mass in the FL2 detector following $280 \mathrm{~nm}$ excitation. So while quinine is not a biological molecule we expect to exist in any abundance in the atmosphere (unlike NADH), its stability and similar emission properties to NADH make it our calibrant of choice for the FL2 detector.

\subsection{Detection limits}

The development of an absolute calibration method allows us to examine limits of detection for both detectors as a function of gain voltage. As discussed above, we used the FT mode to assess the background noise in each channel, taking three standard deviations above the mean $(\mathrm{FT}+3 \sigma)$ to be the threshold for designating a particle as "fluorescent". This is a relatively conservative threshold for which only $0.3 \%$ of FT observations would be falsely classified as fluorescent. The lower limit of detection, defined as the lowest mass that can be reliably distinguished from background (Armbruster and Pry, 2008), is in this case the mass of fluorophore that would result in signal above this threshold for a particular gain setting. We define the upper LOD as the largest mass that gives signal without saturating the detector. To calculate the lower LOD, we use the intersection of the calibration fit line with $\mathrm{FT}+3 \sigma$. The upper LOD is determined by the intersection of the calibration fit line with the saturating level of the detector. Because of the nonlinearity of the quinine results over the entire particle mass range, the linear fits used to determine the lower LOD as described above were for particle masses less than $1000 \mathrm{fg}$ quinine.

Figure 11 shows "detection bands" for each fluorophore as a function of detector gain voltage. The left axis shows the mass at the lower LOD (bottom of band) and the upper LOD (top of band) as a function of gain for both tryptophan (Fig. 11a) and quinine (Fig. 11b). Dotted lines on Fig. 11 are visual guides that illustrate the boundaries on the detectable range of fluorophore mass. The left axis is split to illustrate the magnitude of changes in the upper and lower LOD with changes in gain setting. Each detection band illustrates the mass range of each calibrant that can be resolved at each gain setting tested in this study.

For both detectors, increasing the gain voltage results in small changes to the lower LOD. For example, the lower LOD of tryptophan in the FL1 detector is $2.18 \mathrm{fg}$ at the low gain setting $(0.632 \mathrm{~V})$ and $2.17 \mathrm{fg}$ at the higher gain setting $(0.747 \mathrm{~V})$. At the same time, however, there is a $\sim 4 \mathrm{x}$ increase in the upper LOD between the two gain settings, with 335 and $79.9 \mathrm{fg}$ tryptophan saturating at 0.632 and $0.747 \mathrm{~V}$, respectively. Similarly for FL2, there is a marginal decrease in the detection limit by using one of the two higher gain settings tested compared to the lowest gain setting $(0.508 \mathrm{~V})$. However, the measurable mass range for this lowest gain setting is far larger. The quinine mass that saturates the detector at the low gain setting is an order of magnitude larger compared to the highest gain setting tested. All lower and upper LOD values for each calibration material can be found in Tables 2 and 3 . 
If the goal is to maximize detection of small amounts of fluorescent material, a higher gain setting may be marginally beneficial. However, the loss of fluorescence intensity information for strongly fluorescent particles at high gain settings is considerable. Perhaps loss of information may not matter to the user if they are employing a binary above-below threshold classification scheme, where the magnitudes of the fluorescence signals are not considered. On the other hand, as in the clustering study performed by Robinson et al. (2013) in which saturating particles had to be excluded from analysis, a lower gain setting is likely optimal in most cases, as the dynamic range of measurable fluorophore mass is much larger. There may be considerable value in analyzing the fluorescence signal magnitudes in ambient data sets, and we recommend running instruments like the WIBS with gain settings that allow for the maximum range of mass determination.

\subsection{Effect of flash lamp intensity on fluorescence signals}

For both of our calibration materials, quinine and tryptophan, as well as fluorescent PSLs, fluorescence magnitudes decreased linearly with increasing optical density (OD) values for the filters used. Optical density is defined in terms of transmission $(T)$, according to $\mathrm{OD}=\log _{10} T$. Figure 10 shows the fluorescence signals from $1.0 \mu \mathrm{m}$ blue fluorescent PSLs (Fluoro-Max ${ }^{\mathrm{TM}}$ B0100, Thermo Scientific; Fremont, CA, USA) as a function of percent transmission of the native Xe flash lamp output. Each data point is the center of a Gaussian function fit to a histogram of FL2 data from the FPSLs following the insertion of a neutral density filter in the lens tube between the lamp and the lens that focuses the lamp light on the particle beam. Experiments with both quinine particles and mixed ammonium sulfate-tryptophan particles also showed this linear relationship in fluorescence magnitudes with transmission. We saw no difference between our original (3-year-old) flash lamp and a brand new flash lamp in the magnitudes of fluorescence signals for any of the materials tested, indicating that they are producing the same amount of excitation power.

Clearly, the fluorescence intensity measured in the WIBS is highly dependent on the output power from the flash lamp. Due to the high sensitivity of the fluorescence on the input excitation energy, it would be desirable to have a direct measurement of the flash power or at least a more robust relative measure of flash power that is not subject to very minor changes in, for example, alignment, as is the fiber-optic sensor in the WIBS-4A. We recommend this as an area of improvement for future models of the instrument. Nonetheless, the fluorescent particles described here are a useful diagnostic tool, as any decrease in flash lamp power with time should be identifiable by decreases in signal from these fluorescence standards.

Using these two materials, we have calibrated the detection sensitivity of a single-particle fluorescence instrument

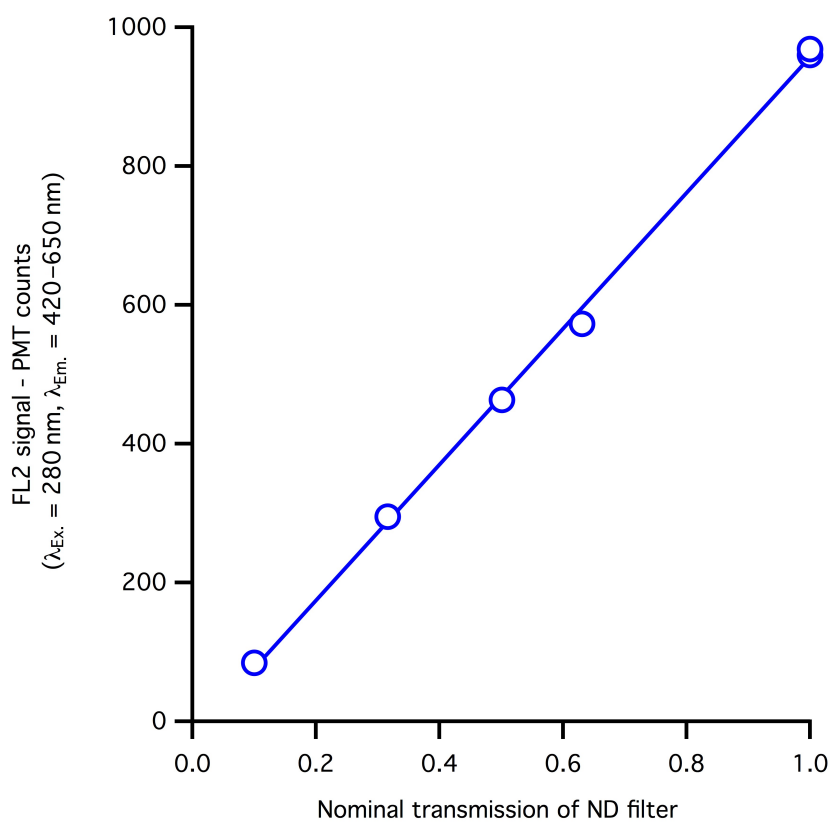

Figure 10. Fluorescence signal magnitudes vs. transmission of xenon flash lamp light. Fluorescence signal magnitudes are the central values of fitted Gaussian functions to histograms of FL2 data from $1 \mu \mathrm{m}$ blue fluorescent PSLs. Nominal transmission values are calculated according to $T=10^{-\mathrm{OD}}$, where $\mathrm{OD}$ is the nominal manufacturer-quoted optical density.

(WIBS-4A) to a given mass of fluorescent material. Importantly, the overall sensitivity derived through these calibration experiments does not decouple the detector performance from the excitation energy input. But, the method allows users to determine how their instrument responds to particles containing a known amount of quinine or tryptophan, and the resulting calibration curves can then be used to quantify, in absolute terms, the fluorescence of, for example, ambient fluorescent particles. Therefore, we propose reporting fluorescence intensities in units of fluorophore equivalent mass (e.g., "quinine equivalent mass" or "Q-units"). If WIBS users report these "T-units" or Q-units after properly assessing the sensitivity of their instruments, measurements of this fluorescence intensity should be directly comparable between instruments. As an example, we quantify the absolute fluorescence of the $1 \mu \mathrm{m}$ blue FPSLs shown in Fig. 10 being equivalent to the fluorescence of $3400 \mathrm{fg}$ quinine (or $3400 \mathrm{Q}$-units).

\section{Conclusions}

We have presented a simple fluorescence calibration technique for single-particle fluorescence spectrometers and demonstrated its utility with a WIBS-4A instrument. We have shown different aerosolized solutions to be fluorescent in the WIBS, stable with time, repeatable across different solution preparations and experiments, and safe to produce, 


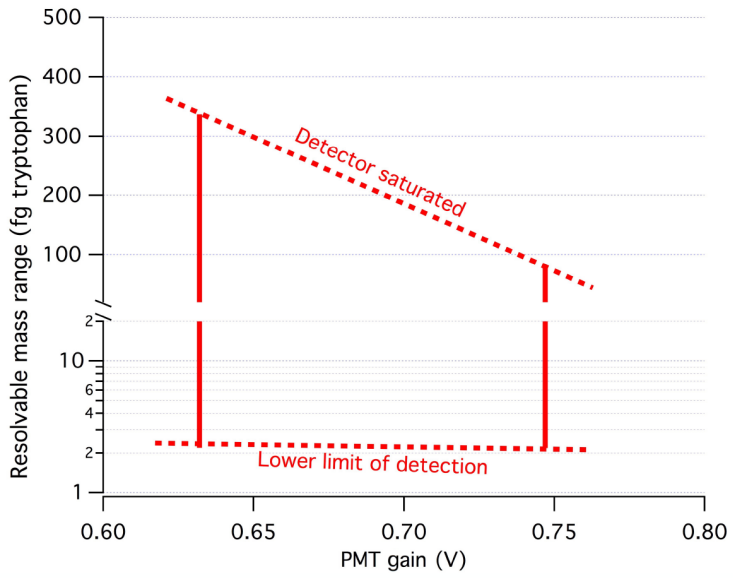

(a)

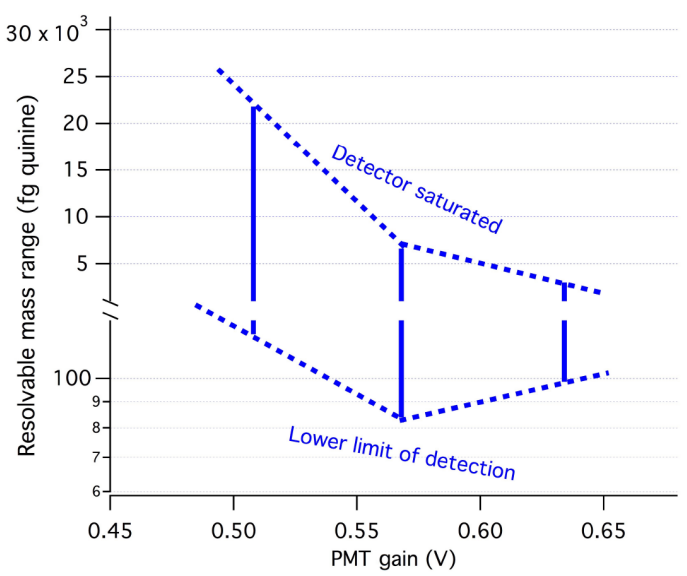

(b)

Figure 11. Detection bands showing the lower limit of detection and the fluorophore mass that saturates the detector as a function of detector gain voltage for (a) the FL1 channel (tryptophan) and (b) the FL2 channel (quinine). Dotted lines are visual guides showing the range of fluorophore mass that is outside of the detection window.

thus meeting our requirements for use as calibration standards. Use of a DMA to select narrow particle size ranges allows for construction of a fluorescence calibration curve that relates the PMT fluorescence signal from individual particles to fluorophore mass. Adopting the calibration curves used in these instruments in sampling studies will improve the ability to directly compare results between instruments and allow users to set the gains on their detectors in a more informed and consistent way. Instituting an absolute fluorescence scale will lead to utilizing fluorescence intensities in ambient fluorescent aerosol studies and to better comparisons between different instruments. We also highlight a need for the WIBS community to better monitor flash lamp power, as the fluorescence signals are in a linear response regime with respect to flash power.

Data availability. Data used in this study can be made available upon request to the author.

Competing interests. The authors declare that they have no conflict of interest.

Acknowledgements. This work was conducted and supported under the NOAA Atmospheric Composition and Climate Program and the NOAA Health of the Atmosphere Program.

Edited by: F. Pope

Reviewed by: P. Williams and one anonymous referee

\section{References}

Armbruster, D. A. and Pry, T.: Limit of blank, limit of detection and limit of quantitation, The Clinical Biochemist Reviews, 29, S49-S52, 2008.

Bhangar, S., Adams, R. I., Pasut, W., Huffman, J. A., Arens, E. A., Taylor, J. W., Bruns, T. D., and Nazaroff, W. W.: Chamber bioaerosol study: human emissions of size-resolved fluorescent biological aerosol particles, Indoor Air, 26, 193-206, 2015.

Chudakov, D. M., Matz, M. V., Lukyanov, S., and Lukyanov, K. A.: Fluorescent Proteins and Their Applications in Imaging Living Cells and Tissues, Physiol. Rev., 90, 1103-1163, 2010.

Crawford, I., Robinson, N. H., Flynn, M. J., Foot, V. E., Gallagher, M. W., Huffman, J. A., Stanley, W. R., and Kaye, P. H.: Characterisation of bioaerosol emissions from a Colorado pine forest: results from the BEACHON-RoMBAS experiment, Atmos. Chem. Phys., 14, 8559-8578, doi:10.5194/acp-14-8559-2014, 2014.

Crawford, I., Ruske, S., Topping, D. O., and Gallagher, M. W.: Evaluation of hierarchical agglomerative cluster analysis methods for discrimination of primary biological aerosol, Atmospheric Measurement Techniques, 8, 4979-4991, 2015.

Crawford, I., Lloyd, G., Herrmann, E., Hoyle, C. R., Bower, K. N., Connolly, P. J., Flynn, M. J., Kaye, P. H., Choularton, T. W., and Gallagher, M. W.: Observations of fluorescent aerosolcloud interactions in the free troposphere at the High-Altitude Research Station Jungfraujoch, Atmos. Chem. Phys., 16, 2273 2284, doi:10.5194/acp-16-2273-2016, 2016.

Creamean, J. M., Suski, K. J., Rosenfeld, D., Cazorla, A., DeMott, P. J., Sullivan, R. C., White, A. B., Ralph, F. M., Minnis, P., Comstock, J. M., Tomlinson, J. M., and Prather, K. A.: Dust and Biological Aerosols from the Sahara and Asia Influence Precipitation in the Western U.S., Science, 339, 1572-1578, 2013.

Després, V. R., Alex Huffman, J., Burrows, S. M., Hoose, C., Safatov, A. S., Buryak, G., Fröhlich-Nowoisky, J., Elbert, W., Andreae, M. O., Pöschl, U., and Jaenicke, R.: Primary biological 
aerosol particles in the atmosphere: a review, Tellus B, 64, 1-58, doi:10.3402/tellusb.v64i0.15598, 2012.

Faris, G. W., Copeland, R. A., Mortelmans, K., and Bronk, B. V.: Spectrally resolved absolute fluorescence cross sections for Bacillus spores, Appl. Optics, 36, 958-967, 1997.

Gabey, A. M., Gallagher, M. W., Whitehead, J., Dorsey, J. R., Kaye, P. H., and Stanley, W. R.: Measurements and comparison of primary biological aerosol above and below a tropical forest canopy using a dual channel fluorescence spectrometer, Atmos. Chem. Phys., 10, 4453-4466, doi:10.5194/acp-10-4453-2010, 2010.

Georges, J., Arnaud, N., and Parise, L.: Limitations arising from optical saturation in fluorescence and thermal lens spectrometries using pulsed laser excitation: Application to the determination of the fluorescence quantum yield of rhodamine 6G, Appl. Spectrosc., 50, 1505-1511, 1996.

Goldberg, J. M., Wissner, R. F., Klein, A. M., and Petersson, E. J.: Thioamide quenching of intrinsic protein fluorescence, Chem. Commun., 48, 1550-1552, 2012.

Greenwood, D. P., Jeys, T. H., Johnson, B., Richardson, J. M., and Shatz, M. P.: Optical Techniques for Detecting and Identifying Biological-Warfare Agents, Proceedings of the IEEE, 97, 971989, 2009.

Hill, S. C., Pinnick, R. G., Niles, S., Pan, Y. L., Holler, S., Chang, R. K., Bottiger, J., Chen, B. T., Orr, C. S., and Feather, G.: Realtime measurement of fluorescence spectra from single airborne biological particles, Field Anal. Chem. Tech., 3, 221-239, 1999.

Kaye, P., Stanley, W. R., Hirst, E., Foot, E. V., Baxter, K. L., and Barrington, S. J.: Single particle multichannel bio-aerosol fluorescence sensor., Opt. Express, 13, 3583-3593, 2005.

Lawkowicz, J. R., Szmacinski, H., Nowaczyk, K., and Johnson, M. L.: Fluorescence Lifetime Imaging of Free and ProteinBound Nadh, P. Natl. Acad. Sci. USA, 89, 1271-1275, 1992.

Morris, C. E., Conen, F., Alex Huffman, J., Phillips, V., Pöschl, U., and Sands, D. C.: Bioprecipitation: a feedback cycle linking Earth history, ecosystem dynamics and land use through biological ice nucleators in the atmosphere, Glob. Change Biol., 20, 341-351, 2013.

Pan, Y.-L., Hartings, J., Pinnick, R. G., Hill, S. C., Halverson, J., and Chang, R. K.: Single-Particle Fluorescence Spectrometer for Ambient Aerosols, Aerosol Sci. Tech., 37, 628-639, 2003.

Pant, D., Tripathi, U. C., Joshi, G. C., Tripathi, H. B., and Pant, D. D.: Photophysics of Doubly-Charged Quinine - Steady-State and Time-Dependent Fluorescence, J. Photoch. Photobio. A, 51, 313-325, 1990.
Perring, A. E., Schwarz, J. P., Baumgardner, D., Hernandez, M. T., Spracklen, D. V., Heald, C. L., Gao, R. S., Kok, G., McMeeking, G. R., McQuaid, J. B., and Fahey, D. W.: Airborne observations of regional variation in fluorescent aerosol across the United States, J. Geophys. Res.-Atmos., 120, 1153-1170, 2015.

Pöschl, U., Martin, S. T., Sinha, B., Chen, Q., and Gunthe, S. S.: Rainforest aerosols as biogenic nuclei of clouds and precipitation in the Amazon, Science, 329, 1513-1516, 2010.

Prussin II, A. J., Garcia, E. B., and Marr, L. C.: Total Concentrations of Virus and Bacteria in Indoor and Outdoor Air, Environmental Science \& Technology Letters, 2, 84-88, 2015.

Robinson, N. H., Allan, J. D., Huffman, J. A., Kaye, P. H., Foot, V. E., and Gallagher, M.: Cluster analysis of WIBS single-particle bioaerosol data, Atmos. Meas. Tech., 6, 337-347, doi:10.5194/amt-6-337-2013, 2013.

Rost, F. W. D.: Fluorescence Microscopy, vol. 2, Cambridge University Press, Cambridge, UK, 1992.

Rover, L. E. A.: Study of NADH Stability Using UltravioletVisible Spectrophotometric Analysis and Factorial Design, Anal. Biochem., 260, 50-55, 1998.

Tobias, H. J., Schafer, M. P., Pitesky, M., Fergenson, D. P., Horn, J., Frank, M., and Gard, E. E.: Bioaerosol Mass Spectrometry for Rapid Detection of Individual Airborne Mycobacterium tuberculosis H37Ra Particles, Appl. Environ. Microb., 71, 6086-6095, 2005.

Toprak, E. and Schnaiter, M.: Fluorescent biological aerosol particles measured with the Waveband Integrated Bioaerosol Sensor WIBS-4: laboratory tests combined with a one year field study, Atmos. Chem. Phys., 13, 225-243, doi:10.5194/acp-13225-2013, 2013.

Wright, T. P., Hader, J. D., McMeeking, G. R., and Petters, M. D.: High Relative Humidity as a Trigger for Widespread Release of Ice Nuclei, Aerosol Sci. Tech., 48, i-v, 2014.

Yu, X., Wang, Z., Zhang, M., Kuhn, U., Xie, Z., Cheng, Y., Pöschl, U., and Su, H.: Ambient measurement of fluorescent aerosol particles with a WIBS in the Yangtze River Delta of China: potential impacts of combustion-related aerosol particles, Atmos. Chem. Phys., 16, 11337-11348, doi:10.5194/acp-16-11337-2016, 2016. 